\title{
Visualization of hepatobiliary excretory function by intravital multiphoton microscopy
}

\section{Yuan Liu}

National Taiwan University

Department of Physics

Taipei 106 Taiwan

\author{
Hsiao-Ching Chen \\ Shu-Mei Yang \\ National Taiwan University Hospital \\ and National Taiwan University \\ College of Medicine \\ Department of Internal Medicine \\ Taipei 100 Taiwan
}

\section{Tzu-Lin Sun}

Wen Lo

National Taiwan University

Department of Physics

Taipei 106 Taiwan

\section{Ling-Ling Chiou \\ Guan Tarn Huang}

National Taiwan University Hospital and National Taiwan University

College of Medicine

Department of Internal Medicine

Taipei 100 Taiwan

\section{Chen-Yuan Dong}

National Taiwan University

Department of Physics

Taipei 106 Taiwan

E-mail: cydong@phys.ntu.edu.tw

\section{Hsuan-Shu Lee}

National Taiwan University

College of Bio-Resources and Agricultural Institute of Biotechnology

Taipei 106 Taiwan and

National Taiwan University Hospital and National Taiwan University

College of Medicine

Department of Internal Medicine

Taipei 100 Taiwan

E-mail: benlee@ha.mc.ntu.edu.tw

\begin{abstract}
Intravital imaging of hepatobiliary excretion is vital for elucidating liver metabolism. In this work, we describe a novel method to observe the intravital dynamics of the uptake, processing, and excretion of an organic anion, 6-carboxyfluorescein diacetate (6-CFDA) in the hepatobiliary system. This is achieved by the use of multiphoton microscopy and an intravital hepatic imaging chamber. The highquality images show sequential uptake and processing of 6-CFDA from the hepatocytes and the subsequent excretion into bile canaliculi within approximately $50 \mathrm{~min}$. This is a promising technique to study intravital hepatic physiology and metabolism. () 2007 Society of PhotoOptical Instrumentation Engineers. [DOI: 10.1117/1.2710237]
\end{abstract}

Keywords: multiphoton microscopy; hepatobiliary excretion function; hepatocytes; bile canaliculi; 6-carboxyfluorescein; intravital imaging.

Paper 06128LRR received May 18, 2006; revised manuscript received Aug. 24, 2006; accepted for publication Aug. 28, 2006; published online Feb. 28, 2007.

\section{Introduction}

One of the major hepatobiliary functions is the uptake, processing, and excretion of a variety of endogenous or exog-

Address all correspondence to Chen-Yuan Dong, Department of Physics, National Taiwan Univ., Department of Physics National Taiwan University, Taipei, Taiwan 106 Taiwan; Tel: +886-2-3366-5155; Fax: +886-2-2363-9984; E-mail: cydong@phys.ntu.edu.tw or Hsuan-Shu Lee, National Taiwan University, Taipei 106, Taiwan; Tel: +886-2-3366-6007; Fax: +886-2-3366-6001; E-mail: benlee@ha.mc.ntu.edu.tw enous organic anions by the hepatocytes. The anions are obtained from the sinusoids and conjugated with glutathione, glucuronate, or sulfate in hepatocytes, and then excreted by an ATP-dependent mechanism into the bile canaliculi through the apical membrane of hepatocytes. ${ }^{1}$ Conventional methods to investigate these processes usually involved the biochemical analyses of the contents in the liver, bile, or other body

1083-3668/2007/12(1)/014014/5/\$25.00 @ 2007 SPIE 


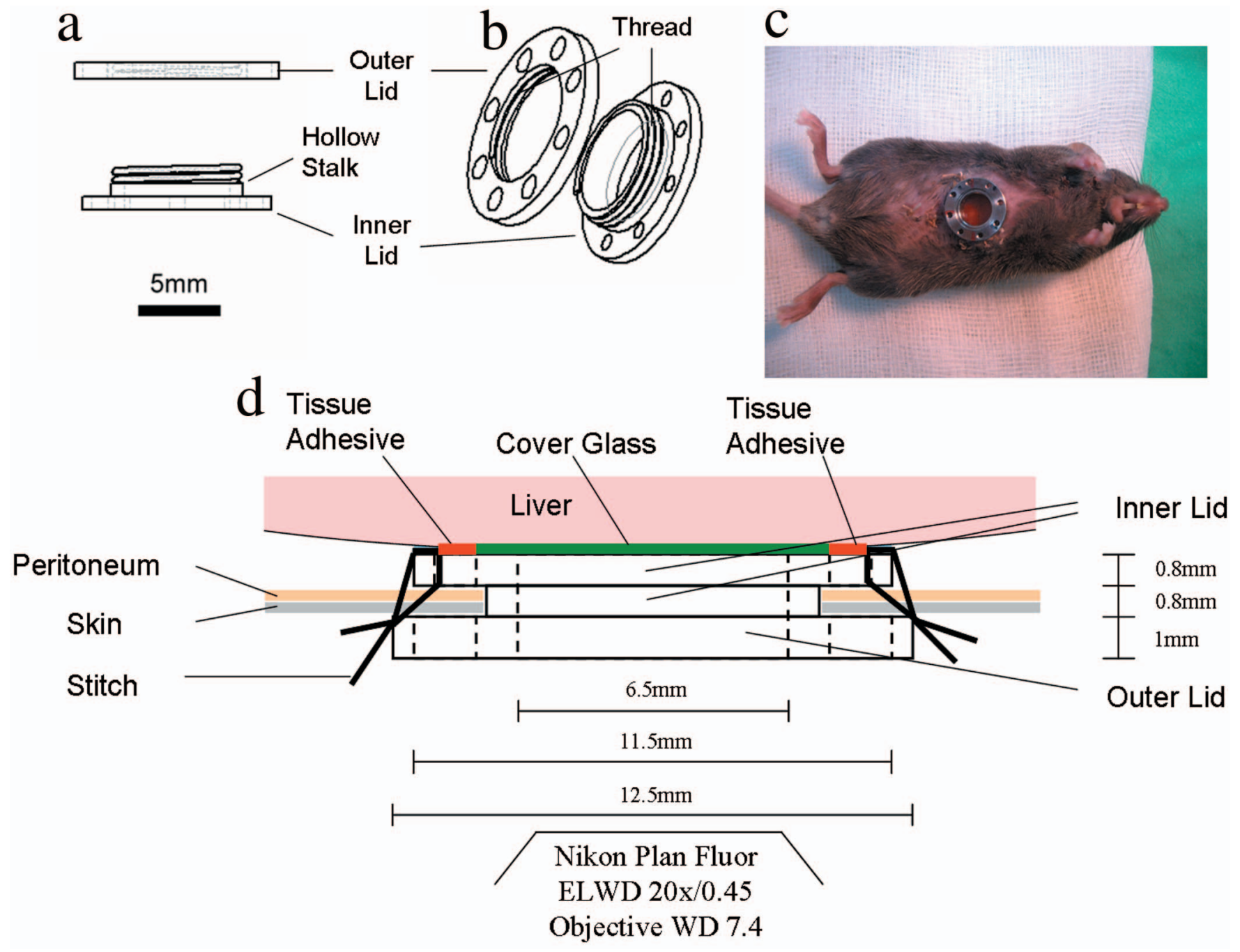

Fig. 1 Illustrations showing the structure of the hepatic imaging chamber and its anatomic position after installation on the mouse abdominal wall. (a) Horizontal and (b) oblique views of the chamber apparatus. (c) A photograph showing a mouse with the device installed on its upper abdominal wall. (d) A diagram depicting the anatomic relationships of the installed device with skin, peritoneum, and the underlying liver.

fluids such as blood or urine. ${ }^{2}$ Real-time imaging of hepatobiliary excretion has been approached on ex-vivo liver tissues by laser confocal microscopy. ${ }^{3}$ However, the ex-vivo model cannot be used to investigate the in-vivo dynamics of hepatic activities. Therefore, the establishment of an intravital imaging system aimed at following these functional processes may be of tremendous value in hepatology.

In this work, we report the design and application of an intravital hepatic imaging chamber on the mouse for the invivo investigation of the hepatobiliary excretory function of 6-CFDA.

\section{Methods}

\subsection{Design of the Intravital Hepatic Imaging Chamber}

Our hepatic imaging chamber is composed of two doughnutshaped titanium rings: the outer and inner lids [Figs. 1(a) and 1(b)]. The outer and inner diameters of the outer lid are 12.5 and $6.5 \mathrm{~mm}$, respectively, with a thickness of $1 \mathrm{~mm}$. For the inner lid, the outer and inner diameters are 11.5 and $6.5 \mathrm{~mm}$, respectively, and it has a thickness of $0.8 \mathrm{~mm}$. Both lids are threaded and can be fastened together by simple screwing.

\subsection{Installation of the Hepatic Imaging Chamber on the Mouse}

C57BL/6 mice beyond 5 weeks of age were anesthetized using intraperitoneal injection of 2-2-2 tribromoethanol at a dose of $0.35 \mathrm{mg} / \mathrm{g}$. The hairs on the abdomen were shaved and a vertical incision of the skin and peritoneum was made. To visualize the sinusoids, the inferior vena cava was exposed for the injection of $10 \mathrm{mg} / \mathrm{mice}$ of rhodamine $\mathrm{B}$ isothiocyanate-dextran 7000 (Sigma, Saint Louis, Missouri). The incision of the lower abdomen was sutured, leaving the upper part open to create a circled wound to position the inner lid of the hepatic imaging chamber inside the abdominal wall. Prior to installing the inner lid, an $8-\mathrm{mm}$ round cover glass was adhered around the outer rim by a polyvinylacetate glue. The cover glass serves as the observation window of intravital hepatic activities. The inner lid was then sutured through the 


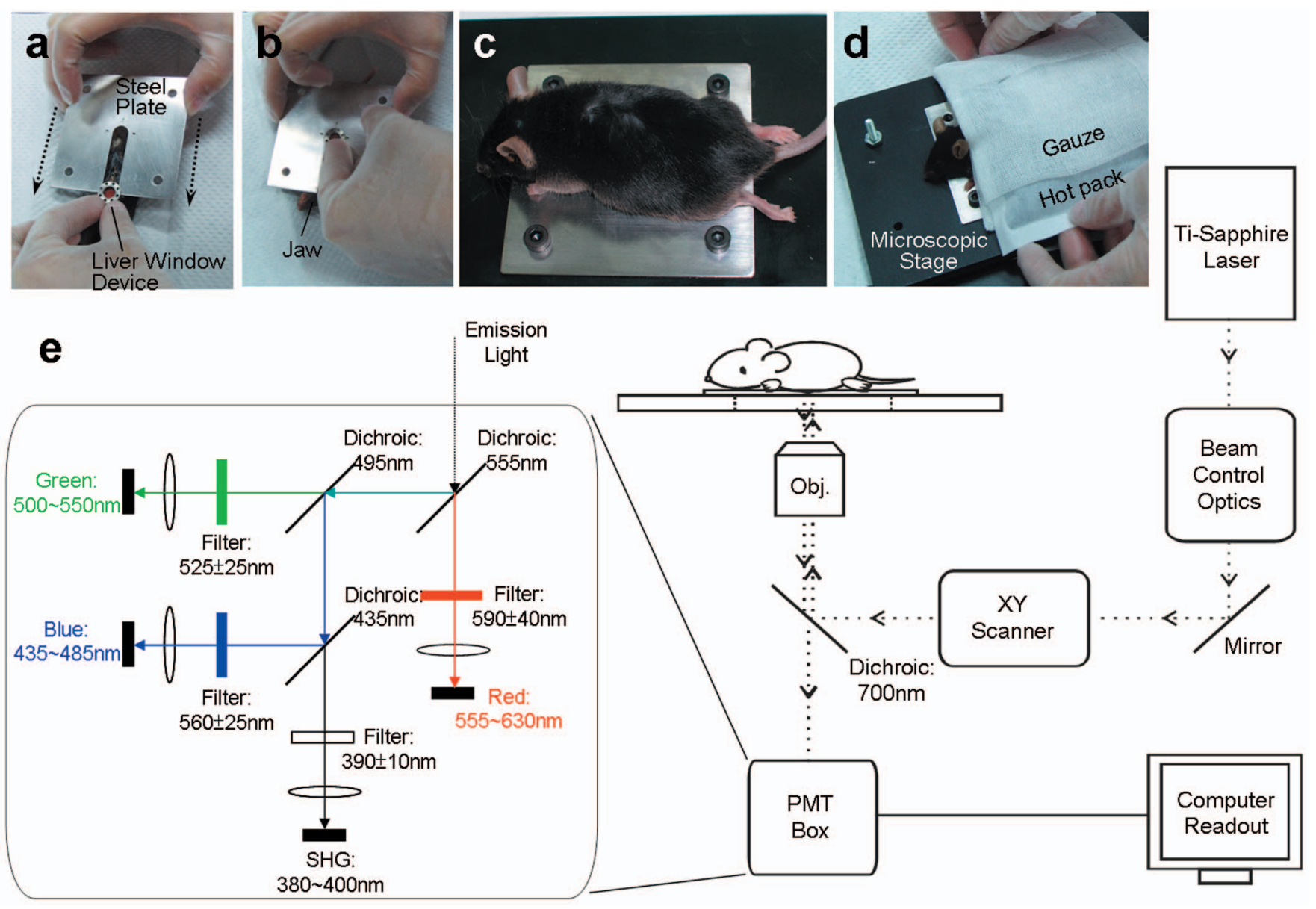

Fig. 2 Accommodation of the mouse on the microscope stage. Fixing of the mouse with its hepatic imaging chamber into the groove of a steel plate was shown in (a), (b), and (c). (d) Fixing the plate onto the microscope stage by screws. (e) A brief schematic of the imaging light path.

small holes on the ring to the skin and peritoneum. To ensure tight contact of the liver surface with the cover glass and to minimize motional artifacts associated with heartbeat and respiration, the adhesive such as tissue adhesive Histoacryl (B. Braun Melsungen AG, Germany) was applied to the edge of the inner lid to adhere the liver to the imaging chamber. For gluing purposes, we tested a number of adhesives. While some such as fibrin did not glue well, others appeared to cause changes in the appearance of the liver surface (not all adhesives were tested under ex-vivo conditions for liver appearance changes). Therefore, the significant criteria of glue selection rest on how well the adhesive can attach the liver to the chamber and the glue's biocompatibility. For comparison purposes, we also acquired images of the hepatic metabolic activities of 6-CFDA using 4011 (for medical device, Henkel Loctite, Rocky Hill, Connecticut) and 406 (instant adhesive, Henkel Loctite). In all cases, the tissue adhesive was not applied to the imaged regions. Finally, the outer lid is attached to the inner lid by screwing [Figs. 1(c) and 1(d)].

\subsection{Accommodation of the Mouse onto the Microscopic Stage}

The positioning of the mouse installed with the hepatic imaging chamber and the subsequent imaging procedures are illustrated in Fig. 2. The mouse was kept in the supine position with the hepatic imaging chamber fitted into the U-shaped groove of a steel plate [Fig. 2(a)], and the plate was slid toward jaw of the mouse and lodged the liver window device to the terminus of the groove [Fig. 2(b)]. The mouse with the steel plate was then placed on the microscopic stage of an inverted microscope (TE2000, Nikon, Japan) by screwing the plate onto the microscopic stage [Fig. 2(c)]. In this manner, the mouse can be firmly attached for multiphoton imaging purposes. A hot pack between the gauze was put on top of the mouse for warming purposes [Fig. 2(d)].

\subsection{Multiphoton Microscopy}

The multiphoton microscope is illustrated in Fig. 2(e). A titanium-sapphire laser with 780-nm output (Tsunami, Spectra Physics, Mountain View, California) pumped by a diodepumped solid-state laser (Millennia X, Spectra Physics) was used for excitation. The laser was scanned by an $x-y$ mirror scanning system (Model 6220, Cambridge Technology, Cambridge, Massachusetts) and guided toward the modified inverted microscope. The laser was beam expanded and reflected into the back aperture of a long working distance $(7.4 \mathrm{~mm})$ objective (Plan Fluor ELWD 20×, NA 0.45, Nikon) by a primary dichroic mirror (700DCSPXRUV-3p, Chroma Technology, Rockingham, Vermont). The power at the sample was around $21 \mathrm{~mW}$, and sample luminescence 

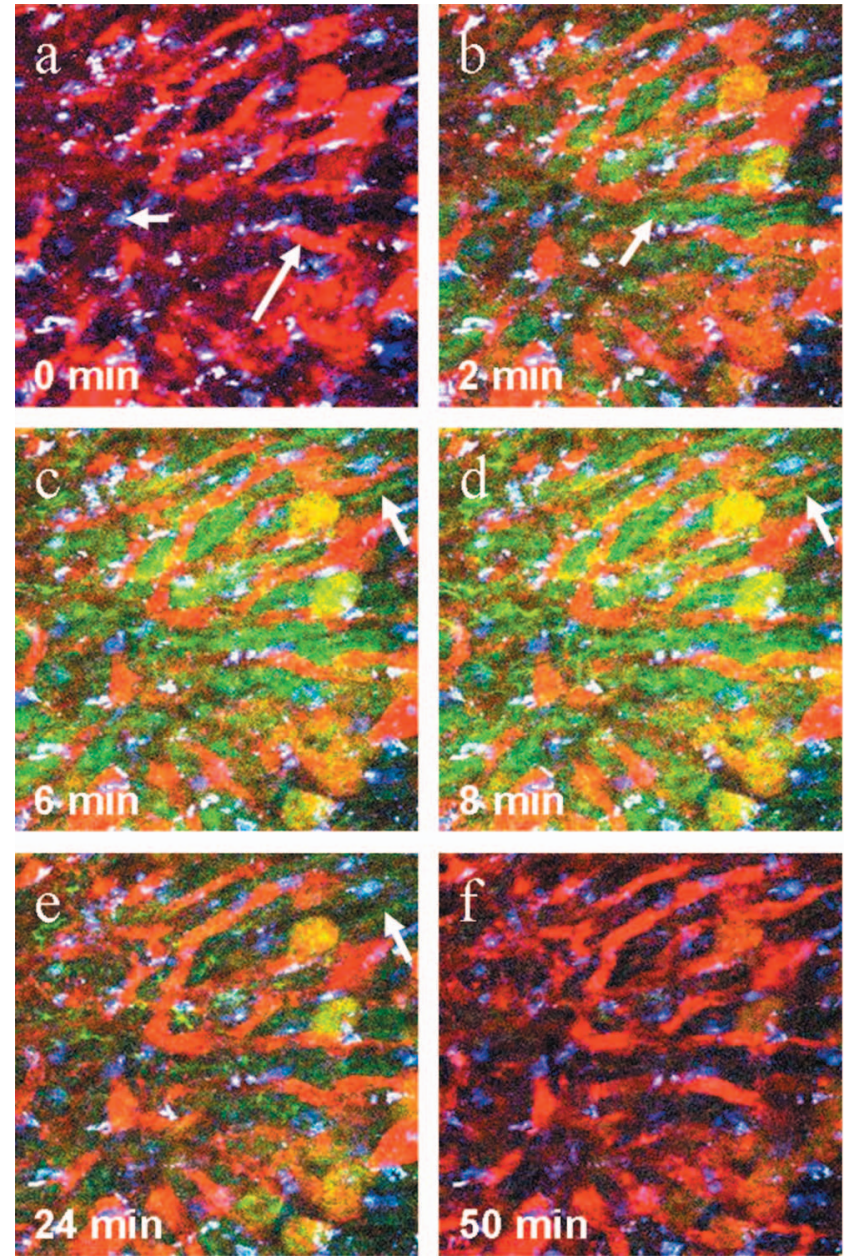

Fig. 3 Representative intravital multiphoton hepatic images acquired using Histoacryl. (a) through ( $\mathrm{f}$ ) are the time-lapsed multiphoton images acquired approximately $30 \mu \mathrm{m}$ below the capsule. Time in minutes indicates period post-6-CFDA injection. Small arrow in (a) indicates the presence of stellate cells (blue), while large arrow indicates sinusoid positions (red). Arrow in (b) indicates hepatocytes in cords emitting CF fluorescence (green), and arrows in (c), (d), and (e) indicate CF fluorescence in bile canaliculi.

was collected in the epi-illuminated or backscattering geometry. After passing through the primary dichroic mirror, the second harmonic generation (SHG) and fluorescence signals are separated into four simultaneous detection channels by secondary dichroic mirrors (435DCXR, 495DCXR, 555DCLP, Chroma Technology) and additional bandpass filters (HQ390/20, HQ460/50, HQ525/50, HQ590/80, Chroma Technology). The detection bandwidths for the SHG, blue, green, and red fluorescence are $390 \pm 10,460 \pm 25,525 \pm 25$, and $590 \pm 40 \mathrm{~nm}$, respectively. In our studies, the SHG signal was collected in the backscattering geometry. Single-photon counting photomultiplier tubes (R7400P, Hamamatsu, Japan) were used as optical detectors. Each optical scan is composed of $256 \times 256$ pixels and took approximately $4 \mathrm{~s}$ to complete. For image processing, we used the software of ImageJ (National Institute of Health, Bethesda, Maryland) and MetaMorph (Universal Imaging Corporation, Downingtown, Pennsylvania). Right before the visualization of hepatobiliary excretory dynamics, we would inject $50 \mu \mathrm{g} / \mathrm{mouse}$ of
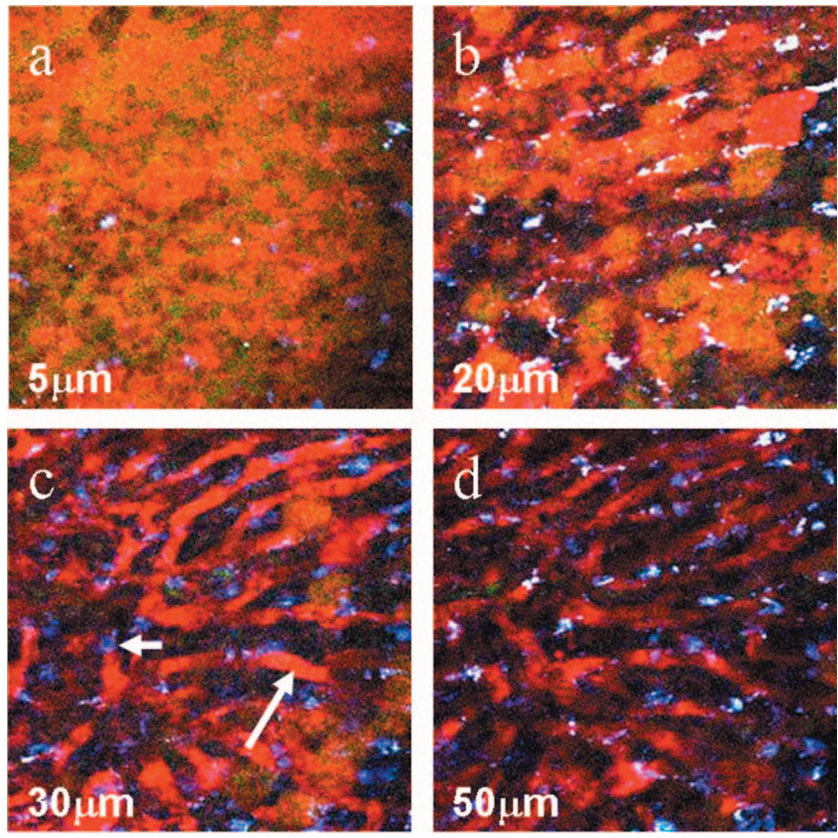

$50 \mu \mathrm{m}$

Fig. 4 Representative intravital multiphoton hepatic images acquired using Histoacryl. Depth-dependent multiphoton images acquired approximately $50 \mathrm{~min}$ after 6-CDFA injection. The stellate cell (small arrow) and sinusoid (large arrow) are clearly visible in (c).

6-carboxyfluorescein diacetate (6-CFDA, Sigma) either through the tail or jugular veins, and began to acquire the serial multiphoton images at 4-s intervals.

The procedures of the animal experiments were approved by the Institutional Laboratory Animal Care Committee of National Taiwan University, College of Medicine.

\section{Results}

Figures 3 and 4 shows the representative multiphoton images acquired using the tissue adhesive Histoacryl. The depth for imaging hepatobiliary function was set at approximately $30 \mu \mathrm{m}$ below the disappearance of the SHG signals from the capsule. Upon injection of 6-CFDA through the jugular vein, the sinusoids already demarcated by the red fluorescence. In addition, the scattered intensely fluorescent spots (5 to $15 \mu \mathrm{m})$ on the margins of the sinusoids were visible [Fig. 3(a)]. These structures are identified as the stellate cells that contain vitamin A emitting strong autofluorescence. ${ }^{4}$ Following 6-CFDA injection, green fluorescence starts to increase in the hepatic cords between the sinusoids and peaks at the 8-min point [Figs. 3(b)-3(d)]. The green fluorescence intensity then starts to decrease and almost reaches the background level at approximately 50-min post-6-CFDA injection [Fig. 3(f)].

Soon after finishing the time-course observations at approximately 50-min post-6-CFDA injection, we imaged the same microscopic field at different depths of 5, 20,30, and $50 \mu \mathrm{m}$ [Figs. 4(a)-4(d)]. The green fluorescence visible in the bile canaculi at earlier time points is no longer apparent. This observation is consistent with the fact that the uptake, processing, and excretion of 6-CFDA is now complete. 


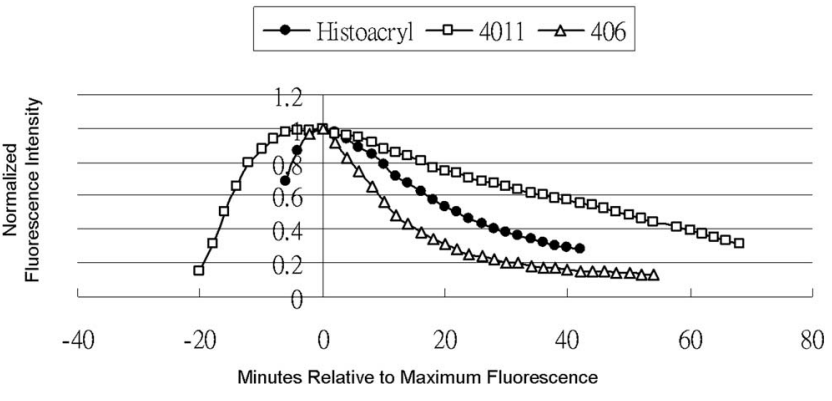

Fig. 5 Measured normalized fluorescence intensity profiles using the glues of Histoacryl, 4011 (for medical device, Henkel Loctite), and 406 (instant adhesive, Henkel, Loctite).

The kinetics of the mean green fluorescence intensity of the liver is shown in Fig. 5. Also shown are the intensity profiles (6-CFDA jugular vein injection) acquired using the adhesives of 4011 and 406. As the figure demonstrates, although the intensity profiles obtained using the three adhesives show different temporal dependence, hepatic metabolism of 6-CFDA is mostly complete at the 50-min mark. Since we only applied the adhesives at the chamber edge, we feel that the effects of glue on hepatic metabolism are minimal. In addition, since the intensity profile of 406 decayed the fastest, the biocompatibility of the glues appear not to be a factor in affecting the hepatic metabolic activities of 6-CFDA. Therefore, we feel that the difference in measured intensity profiles may be due to variations in the individual mouse metabolism and the variations in 6-CFDA injection procedures.

\section{Discussion}

Multiphoton microscopy offers several advantages in the intravital imaging of living tissues. It is superior to videomicroscopy ${ }^{5}$ in its high depth-discrimination nature and also in providing simultaneous multicolor observation of tissue specimens. Multiphoton imaging is also superior to confocal microscopy due to the increased imaging penetration depth and markedly reduced phototoxicity. ${ }^{6}$ In addition, SHG microscopy can also be used to detect collagen fibers in the tissues without the addition of fluorophores. ${ }^{7}$ Intravital multiphoton microscopy has been used in the investigations of renal physiological and pathological processes, ${ }^{8}$ but to the best of our knowledge, it has never been applied in the hepatology field. In this work, we have successfully demonstrated the monitoring of hepatic metabolic activities by the use of multiphoton microscopy and an intravital hepatic imaging chamber.

During the course of this work, we found that the vibration of liver tissues caused by respiration and heartbeat can be minimized by gluing the edges of the inner lid of the hepatic imaging chamber to the underlying liver with tissue adhesive. The adhesive-free regions of the liver were used for imaging purposes. The preadministered rhodamine dextran stays in circulation for as long as $5 \mathrm{~h}$ and thus the sinusoids and venules in the liver are well demarcated by its red fluorescence. 6-CFDA can be taken up by the hepatocytes and then be hy- drolyzed by esterase into carboxyfluorescein (CF), which would emit at $517 \mathrm{~nm}$. ${ }^{9}$ After additional hepatocyte processing, $\mathrm{CF}$ is then excreted into bile. ${ }^{10}$ Our data clearly showed the sequential illumination by $\mathrm{CF}$ of hepatocytes and then bile canaliculi, indicating the hepatobiliary excretion of this organic anion. After the microscopic examination, the animals might, carrying the hepatic imaging chembron their abdomen, live as usual, needing no restriction on activities.

These results support the use of our methodology to be a powerful technique to investigate the hepatobiliary excretory functions in diseases, such as extrahepatic and intrahepatic cholestasis. In addition, hepatic metabolism of chemicals and drugs over an extended period of several days may be investigated with our approach. The application of imaging over a large tissue area may also open a way to observe acute or chronic liver injuries, or follow targeted cell migration and proliferation in complex liver environments.

\section{Acknowledgments}

This work was supported by grants from National Science Councils (NSC 95-3112-B-002-018), Executive Yuan, Taiwan, and was completed using the Optical Molecular Imaging Microscopy Core Facility (A5)of the National Research project for Genomic Medicine.

\section{References}

1. M. Trauner, P. J. Meier, and J. L. Boyer, "Mechanisms of diseasemolecular pathogenesis of cholestasis," N. Engl. J. Med. 339, 12171227 (1998).

2. M. Huber, A. Guhlmann, P. L. M. Jansen, and D. Keppler, "Hereditary defect of hepatobiliary cysteinyl leukotriene elimination in mutant rats with defective hepatic anion excretion," Hepatology (Philadelphia, PA, U. S.) 7, 224-228 (1987).

3. A. Kudo, S. Kashiwagi, M. Kajimura, Y. Yoshimura, K. Uchida, S. Arii, and M. Suematsu, "Kupffer cells alter organic anion transport through multidrug resistance protein 2 in the post-cold ischemic rat liver," Hepatology (Philadelphia, PA, U. S.) 39, 1099-1109 (2004).

4. H. S. Lee, Y. Liu, H. C. Chen, L. L. Chiou, G. T. Huang, W. Lo, and C. Y. Dong, "Optical biopsy of liver fibrosis by use of multiphoton microscopy," Opt. Lett. 29(22), 2614-2616 (2004).

5. I. C. MacDonald, A. C. Groom, and A. F. Chambers, "Cancer spread and micrometastasis development: quantitative approaches for in vivo models," BioEssays 24, 885-893 (2002).

6. P. T. C. So, C. Y. Dong, B. R. Masters, and K. M. Berland, "Twophoton excitation fluorescence microscopy," Annu. Rev. Biomed. Eng. 2, 399-429 (2000).

7. S. W. Teng, H. Y. Tan, J. L. Peng, H. H. Lin, K. H. Kim, W. Lo, Y. Sun, W. C. Lin, S. J. Lin, S. H. Jee, P. T. C. So, and C. Y. Dong, "Multiphoton autofluorescence and second-harmonic generation imaging of the ex vivo porcine eye," Invest. Ophthalmol. Visual Sci. 47, 1216-1224 (2006).

8. B. A. Molitoris and R. M. Sandoval, "Intravital multiphoton microscopy of dynamic renal processes," Am. J. Physiol. 288, F1084-1089 (2005).

9. P. Breeuwer, J. L. Drocourt, N. Bunschoten, M. H. Zwietering, F. M. Rombouts, and T. Abee, "Characterization of uptake and hydrolysis of fluorescein diacetate and carboxy fluorescein diacetate by intracellular esterases in saccharomyces-cerevisiae which result in accumulation of fluorescenct product," Appl. Environ. Microbiol. 61, 16141619 (1995).

10. T. Cantz, A. T. Nies, M. Brom, A. F. Hofmann, and D. Keppler, "MRP2, a human conjugate export pump, is present and transports fluo 3 into apical vacuoles of Hep G2 cells," Am. J. Physiol. 278, G522-531 (2000). 